\title{
Interference Suppression Consisting of Pre-distortion Filtering and Selective Transmit Diversity
}

\author{
Mário Marques da Silva • Américo M. C. Correia • \\ Rui Dinis
}

Published online: 9 February 2008

(C) Springer Science+Business Media, LLC. 2008

\begin{abstract}
In this paper we present and evaluate a pre-distortion (PD) scheme for the downlink of Wideband-code division multiple access (W-CDMA) systems considering a Rake in the receiver in order to exploit multipath diversity. Its performance is compared with schemes without the PD at the base station (BS), and combined with a transmit diversity (TD) scheme to improve the performance by exploiting spatial diversity. It is shown that the proposed PD scheme allows receivers (Mobile Stations) with very low complexity, contrarily to the case where a post-processing multi-user detector (MUD) approach is adopted to improve the performance. Therefore, the proposed PD scheme can be seen as an alternative to post-processing schemes.
\end{abstract}

Keywords W-CDMA · Pre-processing - Selective transmit diversity · Frequency-selective channels $\cdot$ Downlink transmission

\section{Introduction}

Emergent services are demanding higher data rates, especially in the downlink. Widebandcode division multiple access (W-CMDA) is a proven technology to reach this desiderate in cellular communications. To obtain this, it is important to find schemes able to reduce the effects of fading and exploit diversity, as well as to reduce inter-path interference (IPI) and multiple access interference (MAI). When in the presence of frequency selective fading, different symbols suffer from interference from each other, whose effect is usually known as intersymbol interference (ISI), which tends to increase with the increase in the used

M. Marques da Silva $(\varangle) \cdot$ A. M. C. Correia

Instituto de Telecomunicações, IST, Torre Norte 9.26, Av. Rovisco Pais, 1049-001 Lisboa, Portugal e-mail: marques.silva@ieee.org

A. M. C. Correia

ADETTI, IST, Torre Norte 9.26, Av. Rovisco Pais, 1049-001 Lisboa, Portugal

R. Dinis

Instituto de Sistemas e Robótica, IST, Torre Norte 9.26, Av. Rovisco Pais, 1049-001 Lisboa, Portugal 
bandwidth. In case of spread spectrum signals, a multipath environment also originates some level of self-interference. In fact, the replicas of the transmitted signal are not synchronized with the receiver's matched filter, which leads to loss of the orthogonality as well as ISI. In case of W-CDMA, this is also called IPI or self-MAI. When there is more than one user, the multipath channel originates MAI (in this case, inter-users MAI), even for synchronized networks with orthogonal spreading sequences. The Rake receiver, usually considered for W-CDMA systems, takes advantage of the multipath channel to provide multipath diversity (Rake stands for coherent combination of multipath components).

Much research has been undertaken in the area of the multi-user detectors (MUDs) [1,2] for W-CDMA technology. Optimal MUD, usually known as Maximum Likelihood Sequence Detector, are too complex for practical application. Its complexity increases exponentially with the increase of the number of users. On the other hand, sub-optimal MUD has a complexity that increases linearly with the increase of the number of users. The latter can take two different forms: linear MUD (e.g., Decorrelating [3], minimum mean square error (MMSE)) [4] and subtractive MUD (e.g., Successive Interference Cancellation and Parallel Interference Cancellation [1,4]). Linear sub-optimal detectors apply a linear transformation to the bank output of the conventional detectors, in order to decrease the level of MAI seen by each user. Nevertheless, a MUD is normally employed at the BS (uplink) where there is enough power processing capability and where it is easier to know/estimate the uplink channel impulse response (CIR) and the spreading and scrambling sequences of interfering users. Such power processing capability is normally not available at the mobile station (MS) side, being for this reason important to employ alternative schemes to improve the performance.

The trend of the mobile cellular communications is to become more and more asynchronous with bottleneck in the downlink, being the uplink not so demanding in terms of throughput requirements. This is useful for services like Web browsing, Data Base Access, Multimedia Streaming, etc. To reach this desiderate, it is important that the complexity associated to the high downlink traffic is transferred as much as possible from the MS into the $\mathrm{BS}$, where the power processing and electrical power available is higher, in order to combat the several sources of interference.

Since conventional detection techniques for pre-processing systems are normally based on the assumption of a flat propagation channel, they are usually employed for modulations as the orthogonal frequency division multiplexing (OFDM) schemes, which have a flat fading channel behavior at the sub-carrier level. Its extension to W-CDMA systems is not straightforward (due to severely time-dispersive channels), especially when only a Rake is employed at the receiver to exploit multipath diversity [5].

When the downlink CIR information is available at the BS, the use of pre-processing becomes an option, which allows simpler MS receiver implementations (and, eventually better performances). By reducing the signal processing requirements at the MS, the battery drainage can be reduced and terminal cost can be decreased, key aspects in the MS design.

In [6], the authors considered a synchronous multiuser W-CDMA system and developed a pre-processing scheme using the MMSE algorithm, considering a Rake in the receiver. The proposed algorithm by these authors did not take ISI into account.

In [5], the author developed a pre-processing scheme for the downlink of a W-CDMA system, but considering a single decorrelator in the receiver. This approach presents an important disadvantage, as this receiver does not allow multipath diversity exploitation, which is one of the most important properties of W-CDMA signals. In order to exploit the main advantage of W-CDMA, i.e. to exploit multipath diversity, a Rake receiver is normally considered, as described by the Third Generation Partnership Project (3GPP) [7,8]. A wideband channel enables the receiver to resolve a large number of multipath components. With multipath 
diversity, the independent signal copies are sampled in time domain, with the assumption that signals arriving at different delays do not fully correlate.

In [9] the authors proposed a pre-processing scheme using the minimum bit error rate (BER) method for Zero-Forcing equalizer at the receiver. This scheme requires a complex receiver which demands an increase of complexity from the MS.

The contribution of this paper relies on the introduction of a new pre-processing scheme that allows the transfer of the downlink complexity from the receiver (MS) into the transmitter (BS), while keeping the ability to exploit the multipath diversity typical of W-CDMA frequency-selective fading channels, by considering a Rake in the receiver, as considered by 3GPP. The current work was developed based on the work presented in [5], but modifying the pre-distortion (PD) processing, as a Rake is considered at the receiver side. This pre-processing scheme (for the sake of simplicity also called, in this paper, PD) is compared with the conventional scheme without pre-processing and combined with the selective transmit diversity (STD) [10,11], which is a specific TD scheme, as depicted in Fig. 1.

With the aid of pre-distorting the signals to be transmitted by the BS, the orthogonality between the signals seen by the different users can be improved. The proposed system can be seen as a simple alternative to beamforming or multiple input multiple output (MIMO) systems, presenting a much lower MS complexity, without any increase in physical dimensions, and thus, with an easier practical implementation.

This paper is structured as follows: Sect. 2 reviews the two diversity techniques under consideration (STD and Rake receiver); Sect. 3 presents the system model for the proposed PD scheme; Sect. 4 presents the performance results and analysis for the studied schemes in several different environments; the key findings of this paper are then summarized in Sect. 5.

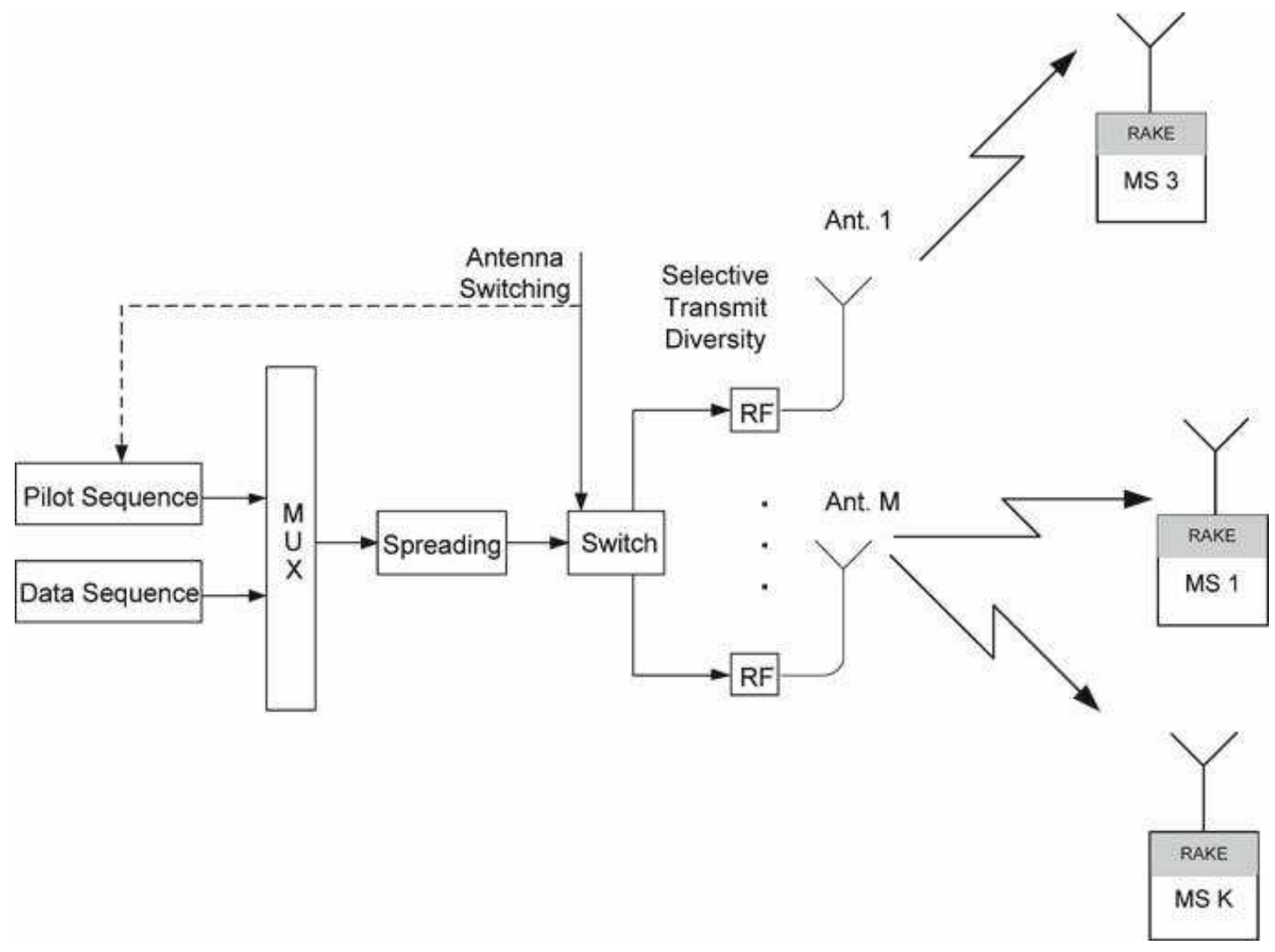

Fig. 1 Selective transmit diversity 


\section{Considered Diversity Techniques}

\subsection{The Selective Transmit Diversity (STD)}

A significant effort has been devoted in 3GPP to develop efficient transmit diversity (TD) solutions to enhance downlink capacity. TD schemes [10] are also applicable for terminals with a single receive antenna, retaining the complexity at the BS. In typical frequency or time diversity the same signal is transmitted over different carrier frequencies or time slots. This results in a decrease in the number of bits per Hertz and a consequent loss in spectral efficiency. To maintain a high spectral efficiency, frequency or time diversity should be based on the transmission of different information symbols over the different frequencies or time slots.

In the case of the downlink, the STD scheme [11] has a low rate feedback link from the receiver (MS) informing the transmitter (BS) which antenna should be used for transmission. Then, the transmitter has link quality information about the several antenna links and it transmits a single information stream over the best antenna. Assuming that the time interval between switching of Time Division Duplex systems is small enough, knowledge about CIR at the transmitter side can be considered available. This assumption is made throughout this paper.

The mix of the PD with the STD can be seen as a composite scheme where the MAI reduction is performed jointly with the exploitation of TD, without additional MS receiver complexity. It is worth noting that the RAKE receiver is not changed by considering the STD scheme, as its fingers are tuned to the multipaths of the channel associated to the selected transmit antenna. Both the PD and the STD schemes refer to the transmitter (i.e., to the BS), and therefore, do not have implications in the receivers' complexity. The TD may also be seen as a pre-processing scheme, as most of the processing is performed at the BS side.

\subsection{The Rake Receiver}

In a multipath channel, the original transmitted signal is reflected from obstacles such as buildings and mountains, and the receiver sees several copies of the signal with different delays. If signals arrive more than one chip apart from each other, the Rake receiver can resolve them. From each multipath signal's point of view, other multipath signals can be regarded as interference and is partially suppressed by the processing gain. However, a further benefit is obtained if the resolved multipath signals are combined using a Rake receiver. Therefore, the signal waveform of W-CDMA signals facilitates the utilization of multipath diversity. In the case of single-path, multipath diversity provided by the Rake receiver would not bring any advantage, and the channel would be flat fading.

A diagram of a Rake receiver is depicted in Fig. 2, which consists of a bank of fingers, each correlating to a different delayed replica of the received signal, followed by a combiner.

After despreading by the decorrelators, the signals are combined using, usually, the maximum ratio combining (MRC) algorithm. ${ }^{1}$ Since the received multipath signals present uncorrelated fadings, diversity is achieved, and thus, performance is improved. After spreading and modulation in the transmitter, the signal is passed through a multipath channel, which can be modelled by a tapped delay line. In the multipath channel there are $L$ multipath components with different delays $\left(T_{1}, T_{2}, \ldots, T_{L}\right)$, attenuation factor $\left(\alpha_{1}, \alpha_{2}, \ldots, \alpha_{L}\right)$ and phase compo-

${ }^{1}$ An MRC combining is considered in the current description. Equal gain or selective combiner tends to achieve poorer performance. 


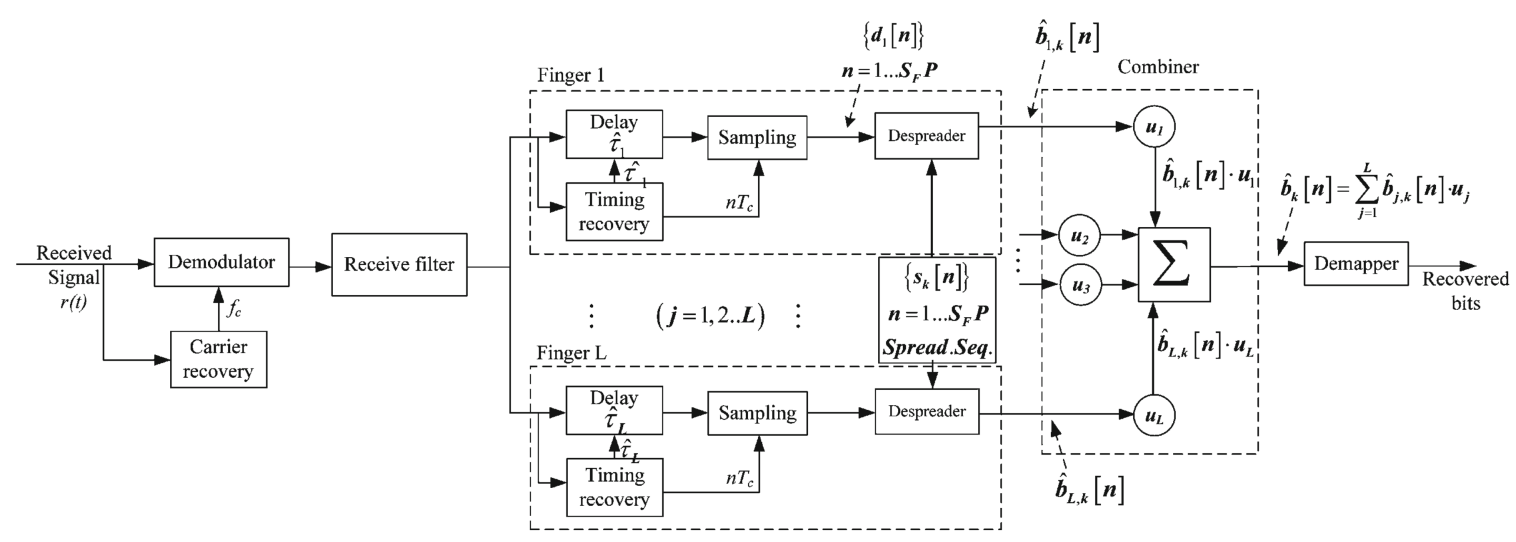

Fig. 2 Diagram of a Rake receiver

nents $\left(\theta_{1}, \theta_{2}, \ldots, \theta_{L}\right)$, each corresponding to a different propagation path. The Rake receiver has, ideally, a different finger for each multipath component. In each finger, the received signal is correlated by the spreading sequence, which is previously time-aligned with the delay of the corresponding multipath signal. After despreading, each captured multipath signal (in each $j$ th finger of the Rake) is weighted, and combined with the others. Moreover, by considering the MRC algorithm to combine the several multipath signals, the signal in each finger is weighted by the complex conjugate $\left(^{*}\right)$ of the corresponding multipath coefficient $\left(u_{j}=\left(\alpha_{j} e^{j \theta}\right)^{*}=\alpha_{j} e^{-j \theta}\right)$ to allow a coherent sum.

Due to the mobile movement, the scattering environment will change, and thus, the delays and attenuation factors will change as well. Therefore, it is necessary to measure the tapped delay line profile and to reallocate Rake fingers whenever the delays have changed by a significant amount. Small-scale changes, less than one chip, are taken care of by a code tracking loop, which tracks the time delay of each multipath signal.

From the diagram depicted in Fig. 2, the $j$ th finger is used to despread the received spread spectrum signal, resulting in the set of despread values:

$$
\hat{b}_{j, k}[n]=\sum_{m=\left[(n-1) \times S_{F}\right]+1}^{n \cdot S_{F}} d_{j}[m] \cdot s_{k}^{*}[m], \quad n=1 \ldots P, j=1 \ldots L,
$$

where $d_{j}[m]$ stands for the $m t h$ sample (sampled at a chip rate) of the output of the receive filter (chip shaping), with a delay corresponding to the $j$ th finger (i.e., to the corresponding multipath) of the Rake receiver. $P$ stands for the length of the symbol sequence under consideration, and $S_{F}$ stands for the spreading factor. Furthermore, $\hat{b}_{j, k}[n]$ corresponds to the $n t h$ estimated transmitted symbol by the $k$ th user, whose estimate is performed using the signal of the $j$ th finger (whose value is based on the corresponding delayed version of the received signal $r\left(t-T_{j}\right)$ ). These despread values are then combined using combining coefficients $u_{1}, u_{2}, \ldots, u_{L}$ to produce a decision statistic.

$$
\hat{b}_{k}[n]=\sum_{j=1}^{L} u_{j} \cdot \hat{b}_{j, k}[n],
$$

where $\hat{b}_{k}[n]$ is subject to a block-decision, typically a hard-decision. 
Assuming that the combiner is based on an MRC, the impulse response of the Rake receiver comes:

$$
h_{R}(T, t)=\sum_{j=1}^{J} \hat{\alpha}_{j}(t) e^{-j \hat{\theta}_{j}(t)} \delta\left(\Delta-t+\hat{T}_{j}\right),
$$

where $\Delta$ is some nominal delay that is required to ensure physical implementation of the Rake, whose value should be higher than the delay corresponding to the last multipath captured by the Rake, corresponding to the last finger. Its frequency response comes

$$
H_{r}(f, t)=\sum_{j=1}^{J} \hat{\alpha}_{j}(t) e^{-j \hat{\theta}_{j}(t)} e^{j 2 \pi f\left(\hat{T}_{j}-\Delta\right)},
$$

where $\widehat{x}$ stands for the estimated value of $x$ and $\hat{\alpha}_{j}(t) e^{-j \hat{\theta}_{j}(t)}$ represents the estimate of the complex conjugate of the multipath coefficient $\hat{\alpha}_{j}(t) e^{j \hat{\theta}_{j}(t)}$.

The fully exploitation of spreaded signals is only achieved by the use of the Rake receiver, because this is the tool to take advantage of multipath diversity. In W-CDMA, the utilization of the spread spectrum with a Rake receiver allows using the frequency selective of the channel as a kind of diversity. For this reason, it is usually stated that the Rake is also a kind of frequency diversity. The resolution of the Rake receiver is the chip period. This is the reason why the resolution is improved when the spreading factor is increased. With a lower chip period, it better discriminates different multipaths, allowing higher order of multipath diversity. On the other hand, when the chip period is too long, the several multipaths are received within the same chip period, providing chip interference and not allowing the corresponding discrimination and diversity.

\section{System Model For The Pre-Distortion Scheme}

Due to the demanding increase of throughputs in the downlink, it is important that the complexity is transferred as much as possible from the mobile station (MS) into the BS, where the power processing and electrical power available is higher in order to combat the several sources of interference. Some previous work has been developed in this area. In [6], a preprocessing scheme was introduced without taking ISI into account, which is an important factor to be considered in the W-CDMA channels. A different pre-processing scheme for the downlink of a W-CDMA system was introduced in [5], but considering a single decorrelator in the receiver (instead of a Rake). In this case, multipath diversity could not be exploited, which is one of the most important properties of W-CDMA signals.

As can be seen from the diagram depicted in Fig. 3, the proposed pre-distortion (PD) processing takes into account the spreading sequences and CIR of the different users present in the cell.

The PD scheme is implemented based on the finite impulse response (FIR) philosophy. The impulse response of the FIR filter for the $q$ th user is defined as

$$
\mathbf{g}_{q}=\left[g_{q}[0] g_{q}[1] \ldots g_{q}\left[L_{g}-1\right]\right]^{T},
$$

where $L_{g}$ stands for the length of the FIR filter. In order to deduct $\mathbf{g}_{q}$, we need first to develop the received signal, after being pre-distorted, spreaded and transmitted by the BS (for the sake of simplicity, a single transmit antenna is here assumed, i.e. no STD) and propagated 


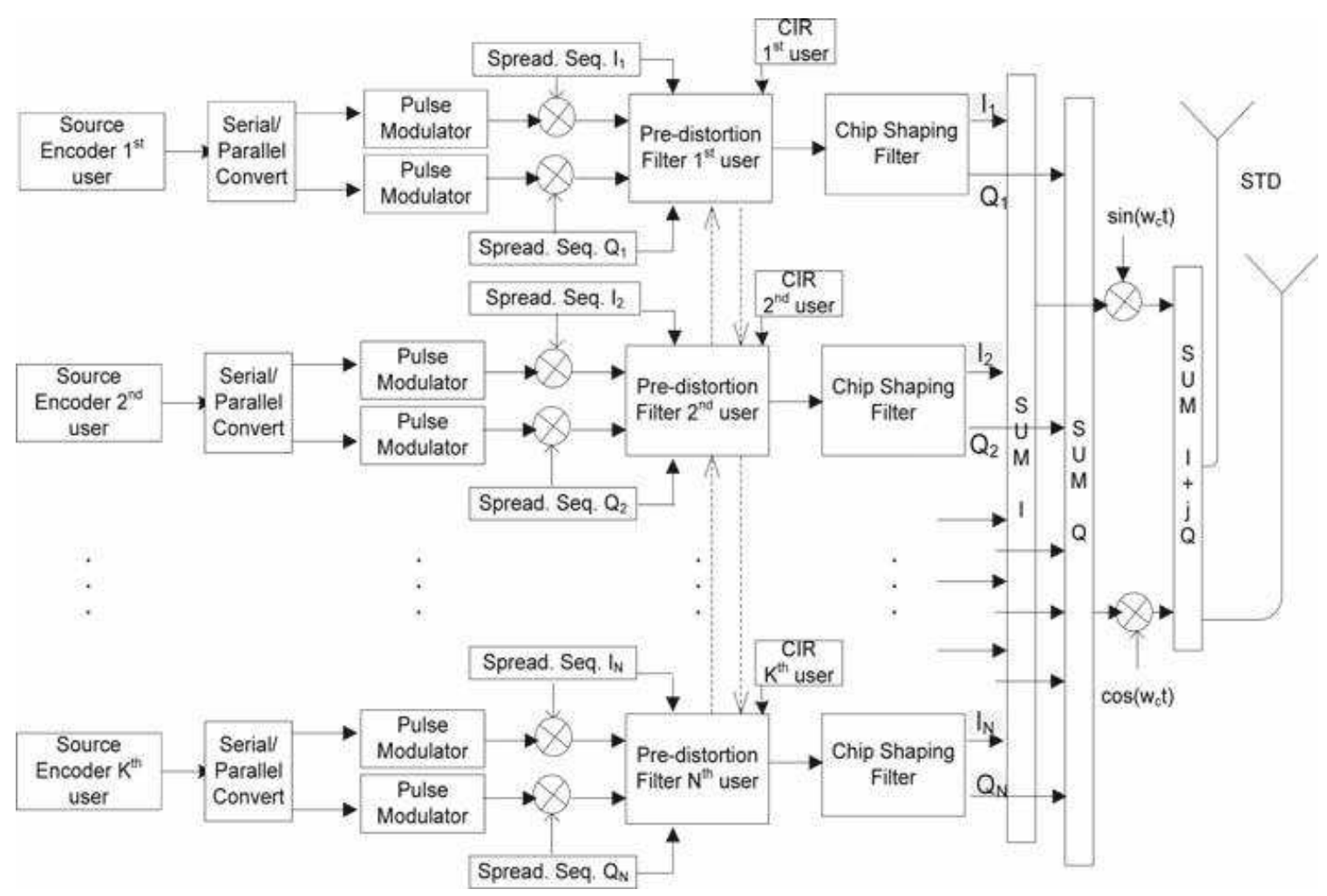

Fig. 3 Scheme of a CDMA-QPSK modulator with combined pre-distortion scheme (STD included)

through a frequency selective fading channel. The received signal at the antenna of the qth MS due to the $k t h$ transmission $c_{q, k}[n]$ is defined, as in [5], by

$$
\begin{aligned}
c_{q, k}[n]= & \mathbf{S}_{k}^{(0)} \mathbf{H}_{q} \mathbf{g}_{k} b_{k}[n] \\
& +\mathbf{S}_{k}^{(-1)} \mathbf{H}_{q} \mathbf{g}_{k} b_{k}[n-1]+\mathbf{S}_{k}^{(1)} \mathbf{H}_{q} \mathbf{g}_{k} b_{k}[n+1]
\end{aligned}
$$

where the $\mathbf{H}_{q}$ matrix, sized $L_{0} \times L_{g}$, is the channel filtering matrix corresponding to a Toeplitz matrix composed of CIR of $q$ th user, defined as $T\left(\mathbf{h}_{q} ; L_{g}\right) .^{2} b_{k}[n]$ is the original symbol to be transmitted. Let's also introduce $L_{0}$, which is defined as $L_{0}=M+L_{g}-1$, being $M$ the maximum delay of the latest multipath under consideration, expressed in chip intervals. The CIR vector of the $q$ th user, which composes the $\mathbf{H}_{q}$ matrix, is defined as $\mathbf{h}_{q}=\left[h_{q}[0] h_{q}[1] \ldots h_{q}[M-1]\right]^{T}$, and where $h_{q}[m](t)=\alpha_{m}(t) e^{j \theta_{m}(t)} \delta\left(t-T_{m}\right)$, as defined for the Rake receiver. For the sake of simplicity the variable $t$ is omitted in the deduction of the PD scheme.

The Toeplitz matrix $\mathbf{S}_{k}^{(0)}[n]$ of size $\left(\mathrm{S}_{\mathrm{F}}+L_{0}-1\right) \times L_{0}$ is defined as $\mathbf{S}_{k}^{(0)}[n]=T\left(\mathbf{s}_{k} ; L_{0}\right)$, where $\mathbf{s}_{k}$ is the spreading sequence of $k$ th user, defined as

$$
\mathbf{s}_{k}=\left[s_{k}[0] s_{k}[1] \ldots s_{k}\left[\mathrm{~S}_{\mathrm{F}}-1\right]\right]^{T}
$$

$\overline{2 \text { The generic Toeplitz matrix }} T(\mathbf{x} ; L)$, with $\mathbf{x}=\left[x_{1} x_{2} \ldots x_{r}\right]$, is defined as $T(\mathbf{x} ; L)=$ $\left[\begin{array}{ccc}x_{1} & & \mathbf{0}_{r+L-1} \\ \cdot & \cdot & x_{1} \\ \cdot & & \cdot \\ x_{r} & & \cdot \\ & \cdot & \\ \mathbf{0}_{r+L-1} & \cdots & x_{r}\end{array}\right]$, being $\mathbf{0}_{r}$ the $r \times 1$ column vector of all zero elements. 
and where $S_{F}$ stands for the spreading factor. In (6) $\mathbf{S}_{k}^{(1)} \mathbf{H}_{q} \mathbf{g}_{k} b_{k}[n+1]$ and $\mathbf{S}_{k}^{(-1)} \mathbf{H}_{q} \mathbf{g}_{k} b_{k}$ $[n-1]$ are IPI vectors where

$$
\begin{aligned}
& \mathbf{S}_{k}^{(-1)}=\left[\begin{array}{llll}
0 & s_{k}\left[\mathrm{~S}_{\mathrm{F}}-1\right] & \cdots & s_{k}\left[\mathrm{~S}_{\mathrm{F}}-L_{0}+1\right] \\
\cdot & \cdot & \cdots & \cdots \\
\cdot & & \cdot & s_{k}\left[\mathrm{~S}_{\mathrm{F}}-1\right] \\
\mathbf{0}_{\mathrm{S}_{\mathrm{F}}} & \mathbf{0}_{\mathrm{S}_{\mathrm{F}}} & \cdots & \mathbf{0}_{\mathrm{S}_{\mathrm{F}}}
\end{array}\right] \\
& \mathbf{S}_{k}^{(1)}=\left[\begin{array}{llll}
\mathbf{0}_{\mathrm{S}_{\mathrm{F}}} & \cdots & \mathbf{0}_{\mathrm{S}_{\mathrm{F}}} & \mathbf{0}_{\mathrm{S}_{\mathrm{F}}} \\
s_{k}[0] & \cdot & \cdots & \cdots \\
\cdot & & \cdot & \cdot \\
s_{k}\left[L_{0}-2\right] & \cdots & s_{k}[0] & 0
\end{array}\right]
\end{aligned}
$$

The received signal at the input of the $q$ th Rake receiver, from all $K$ transmissions, is defined as

$$
c_{q}[n]=\sum_{k=1}^{K} c_{q, k}[n]+z_{q}[n],
$$

being $z_{q}$ the background noise seen by the MS.

Let's define $\mathbf{v}_{q, k}^{(m, i)}$ as the vector with the cross-correlation values between the spreading sequence of some $k$ th interfering user and the shifted version by $m+1$ of the spreading sequence of the qth reference user as

$$
\mathbf{v}_{q, k}^{(m, i)}=\left(\mathbf{S}_{k}^{(i)}\right)^{H} \cdot \mathbf{S}_{q,(m+1)}^{(0)} .
$$

The $i$ th index of $\mathbf{S}_{k}^{(i)}$ can take the values [ $\left.\begin{array}{lll}-1 & 0 & 1\end{array}\right]$, i.e., $\mathbf{S}_{k}^{(0)}[n]=T\left(\mathbf{s}_{k} ; L_{0}\right)$ for $i=0$, and as defined by (9) for $i=-1$ and $i=+1$. The $(m+1)$ index of $\mathbf{S}_{q,(m+1)}^{(0)}$ stands for the the selected column of the spreading sequence of the $q$ th reference user $\left(\mathbf{S}_{q}^{(0)}\right)$, corresponding to the multipath order selected by the Rake finger. Additionally, $\mathbf{w}_{q, k}^{(i)}$ is defined as the $L_{g}$ vector corresponding to the sum of the cross-correlations of $\mathbf{v}_{q, k}^{(m, i)}$, already weighted by the multipath interference $\mathbf{H}_{q}^{(m)}$ (shifted versions of CIR) as

$$
\mathbf{w}_{q, k}^{(i)}=\sum_{m=0}^{M-1}\left(\mathbf{H}_{q}^{(m)}\right)^{H} \mathbf{v}_{q, k}^{(m, i)}
$$

where $\mathbf{H}_{q}^{(m)}$ corresponds to $\mathbf{H}_{q}$ multiplied by the complex conjugate $\left(^{*}\right)$ of the corresponding multipath gain of the $q$ th user, i.e.,

$$
\mathbf{H}_{q}^{(m)}=\mathbf{H}_{q} \cdot h_{q}^{*}[n] .
$$

From (12), it can be understood that it corresponds to the weight of the Maximum Ratio Combining, performed by the corresponding finger of the Rake receiver. The received signal at the output of the Rake receiver of the $q$ th user, after MRC, comes

$$
y_{q}[n]=\sum_{k=1}^{K} \sum_{i=-1}^{1}\left(\mathbf{w}_{q, k}^{(i)}\right)^{H} \mathbf{g}_{k} b_{k}[n+i]+\bar{z}_{q}[n] .
$$


At the $q$ th MS, the interfering signals due to $K$ signals is given by $\sum_{k \neq q}^{K} \sum_{i=-1}^{1}\left(\mathbf{w}_{k, q}^{(i)}\right)^{H} \mathbf{g}_{q} b_{q}$ $[n+i] \quad i=-1,0,1$. The total sum of interfering signals to other MS, including IPI terms due to the $q$ th signal is written as

$$
\begin{aligned}
\boldsymbol{v}_{q}[n]= & \sum_{k \neq q}^{K} \sum_{i=-1}^{1}\left(\mathbf{w}_{k, q}^{(i)}\right)^{H} \mathbf{g}_{q} b_{q}[n] \\
& +\left(\mathbf{w}_{k, q}^{(-1)}\right)^{H} \mathbf{g}_{q} b_{q}[n-1]+\left(\mathbf{w}_{k, q}^{(1)}\right)^{H} \mathbf{g}_{q} b_{q}[n+1] .
\end{aligned}
$$

In order to mitigate the interference to others, the filtering vector $\mathbf{g}_{q}$ should be calculated to minimize $E\left[\left|\boldsymbol{v}_{k}[n]\right|^{2}\right]$, while providing distortionless response to the qth MS (reference user). The optimisation based on the minimum variance of interfering signals to others (MVISO) algorithm is to find $\mathbf{g}_{q}$ such that [5]

$$
\begin{aligned}
& \min _{g_{q}} E\left[\left|\boldsymbol{v}_{q}[n]\right|^{2}\right], \\
& \text { subject to } \mathbf{w}_{q}^{H} \mathbf{g}_{q}=1
\end{aligned}
$$

where $\mathbf{w}_{q}=\mathbf{w}_{q, q}^{(0)}$. There is a closed-form solution for the optimal filtering vector under the MVISO criteria in (15). From (14) it comes:

$$
E\left[\left|\boldsymbol{v}_{q[n]}\right|^{2}\right]=\mathbf{g}_{q}^{H}\left\{\begin{array}{l}
\sum_{k \neq q}\left[\sum_{i=-1}^{1}\left[\mathbf{w}_{k, q}^{(i)} \cdot\left(\mathbf{w}_{k, q}^{(i)}\right)^{H}\right]\right] \\
+\mathbf{w}_{q, q}^{(-1)} \cdot\left(\mathbf{w}_{q, q}^{(-1)}\right)^{H}+\mathbf{w}_{q, q}^{(1)} \cdot\left(\mathbf{w}_{q, q}^{(1)}\right)^{H}
\end{array}\right\} \mathbf{g}_{q} .
$$

Defining

$$
\begin{aligned}
\overline{\mathbf{W}}_{q}= & \sum_{k \neq q}\left[\sum_{i=-1}^{1}\left[\mathbf{w}_{k, q}^{(i)} \cdot\left(\mathbf{w}_{k, q}^{(i)}\right)^{H}\right]\right] \\
& +\mathbf{w}_{q, q}^{(-1)} \cdot\left(\mathbf{w}_{q, q}^{(-1)}\right)^{H}+\mathbf{w}_{q, q}^{(1)} \cdot\left(\mathbf{w}_{q, q}^{(1)}\right)^{H},
\end{aligned}
$$

and using the method of Lagrange multiplier, the optimal vector under the MVISO criteria comes:

$$
\mathbf{g}_{\mathbf{q}}=\frac{\overline{\mathbf{W}}_{q}^{-1} \mathbf{w}_{q}}{\mathbf{w}_{q}^{H} \overline{\mathbf{W}}_{q}^{-1} \mathbf{w}_{q}}, \quad q=1,2, \ldots, K .
$$

This approach corresponds to changing the original users spreading sequences, taking into account the spreading sequences assigned to all users (and their cross-correlation properties) and the several CIRs, in order to minimize the total sum of MAI present in the cell.

\section{Numerical Results}

In this section we present a set of performance results, obtained by simulation, concerning the proposed PD technique, where a Rake in the receiver was always considered. In the simulations the downlink of W-CDMA is considered, using quadrature phase shift keying (QPSK) modulation in a frequency selective Rayleigh fading channel. Both the Pedestrian 
A and Vehicular A of 3GPP [8] were considered. Only uncoded BER performances were computed, with and without the PD scheme, and compared with the 3GPP receiver, which only considers a Rake in the receiver. Walsh-Hadamard spreading sequences were adopted, with spreading factor $S F=16$. The chip period is $T_{C}=1 / 3.84 \mu \mathrm{s}$. The channels associated to different antennas are uncorrelated and perfect channel knowledge is assumed at both the receiver and the transmitter sides. The performances are compared at the link level with BER as performance index, and both fading and interference are jointly studied in the multipath multi-user environment. The BER, using the Monte Carlo simulation, was computed by first sending an extremely long sequence of bits over the described system, and measuring the errors at the receiver side. The BER was then computed by dividing the number of erroneous bits by the total number of transmitted bits.

Different simulations were performed with different multipath environments and spreading factors, where similar performance improvements were achieved. Due to size constraints these results with different conditions are not shown in the current paper. The simulations considered always the PD scheme implemented as a FIR with a dimension six $\left(L_{g}=6\right)$, which leads to better performance than other FIR dimensions (not shown in this paper but part of the research developed). The number of transmit antennas considered to implement the STD was always two. For comparison purposes, this section presents results with "Only Rake" which stands for the system without PD or STD. In this case, the MAI suppression is not achieved, and only multipath diversity is exploited. In addition, results with a "Single user" were also computed and are presented, where the system is only corrupted by ISI, but not by MAI. In this case, a Rake is also considered in the receiver to exploit multipath diversity. This curve is also considered for comparison purposes representing, in some cases, a lower bound.

In Fig. 4 it is shown the performance obtained with 4 users, for the Pedestrian A propagation model of 3GPP. As can be seen, the PD scheme, alone or combined with STD, reaches a

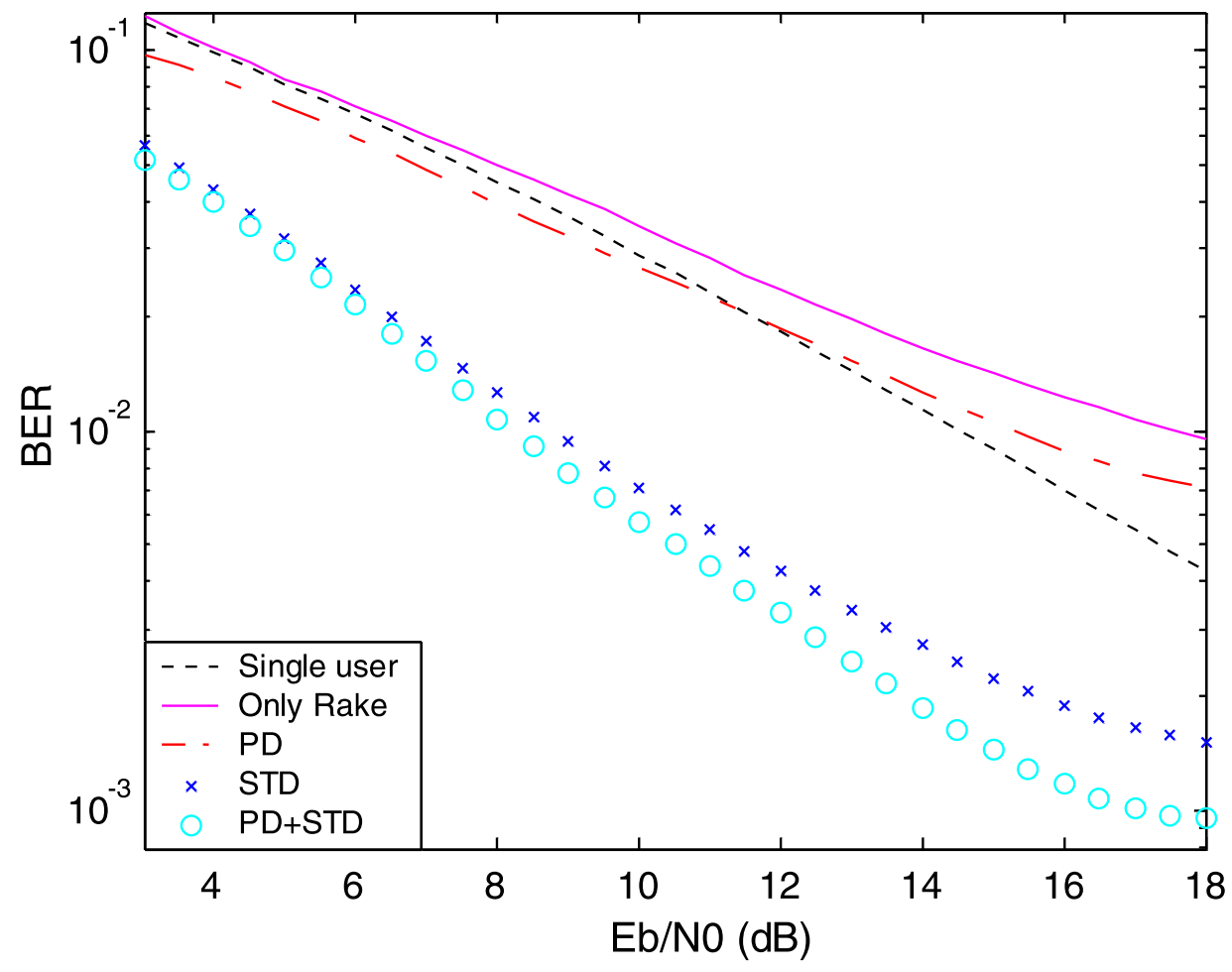

Fig. 4 BER performance for Pedestrian A propagation model with 4 interfering users 
performance improvement over the corresponding schemes without PD (i.e., over the "Only Rake" or STD). This is achieved without any complexity cost at the MS (relating to the 3GPP receiver which considers a Rake) and even the additional complexity at the BS is relatively low. The scheme that achieves the best overall performance is the combination of PD with the STD scheme (PD+STD). While the PD avoids the MAI with a reduction in the crosscorrelation seen by the several MS's, the STD provides an additional gain due to the TD, thus, allowing the receiver to select the antenna that presents better fading conditions, and higher level of multipath diversity.

It is known that the STD normally outperforms the Alamouti Space-Time Coding [12] for the single-path channel profile, while the Alamouti Space-Time Coding normally outperforms the STD for a channel profile closer to the uniform power profile [12]. Since the Pedestrian A propagation model is close to the single path propagation model, it can be seen from Fig. 4 that the performance improvement achieved with the STD over the "Only Rake" is relatively high. It can even be observed that the STD outperforms the "Single user" curve. This occurs because the system with a single user is corrupted by ISI, while the STD exploits $\mathrm{TD}$, even though it has some level of MAI introduced by the 4 users present in the cell.

In all situations, the performance improvement achieved with the PD (with or without STD) is higher for higher levels of $E_{b} / N_{0}$.

The results depicted in Fig. 5 are similar to those obtained from Fig. 4. As can be seen, for 15 users and with the Pedestrian A propagation model of 3GPP, the PD scheme is also able to reach a performance improvement over the "only Rake". As before, the best overall performance is achieved with a combination of the PD with the STD. While the PD avoids the MAI with a reduction in the cross-correlation seen by the several MS's, the STD provides an additional gain due to the TD by allowing the receiver to select the antenna that presents less intense fading, and more intense multipath diversity.

In Fig. 6 it is shown the performance obtained with 4 users, for the Vehicular A propagation model of $3 \mathrm{GPP}$. It can be seen that the performance improvement achieved with the PD

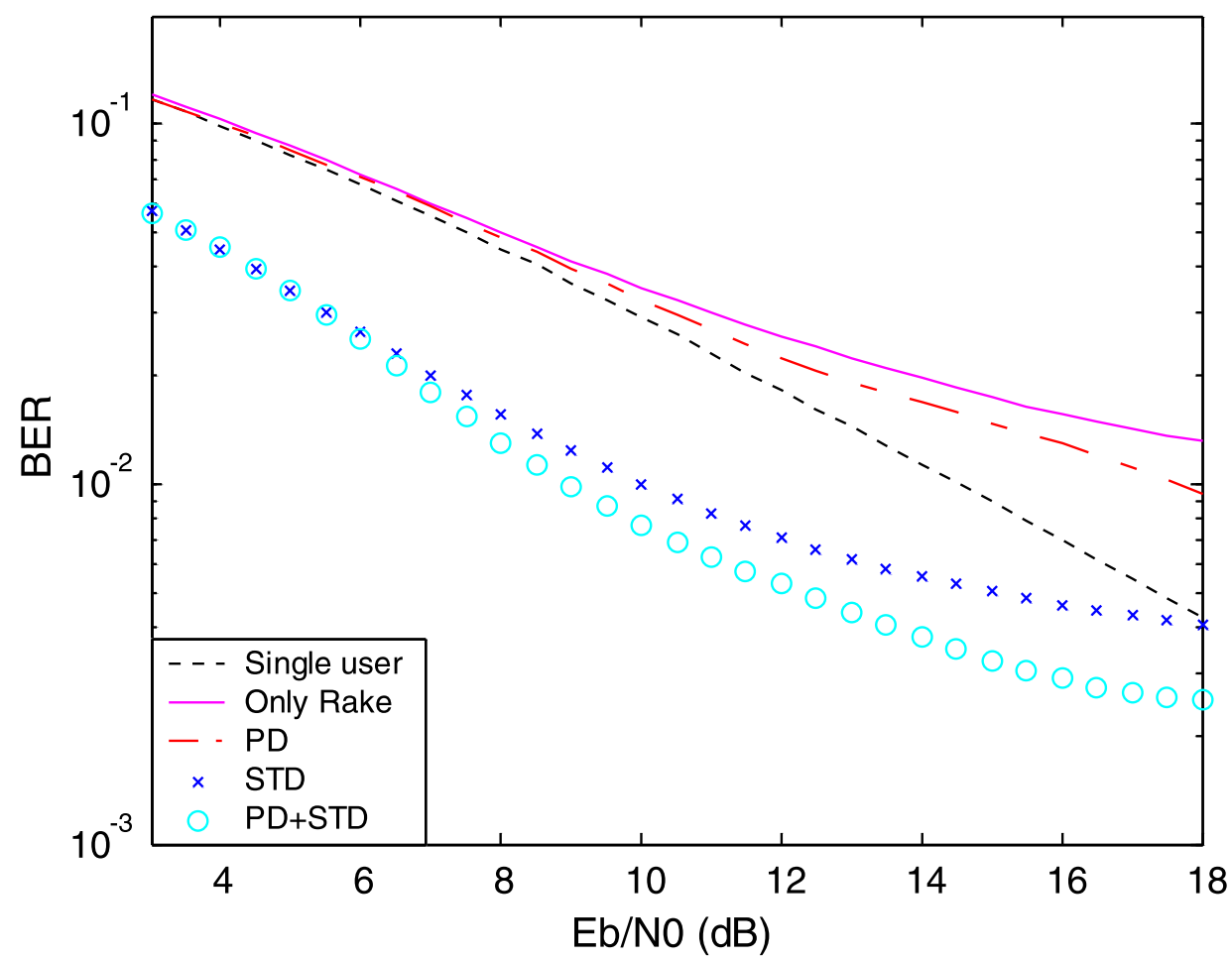

Fig. 5 BER performance for Pedestrian A propagation model with 15 interfering users 


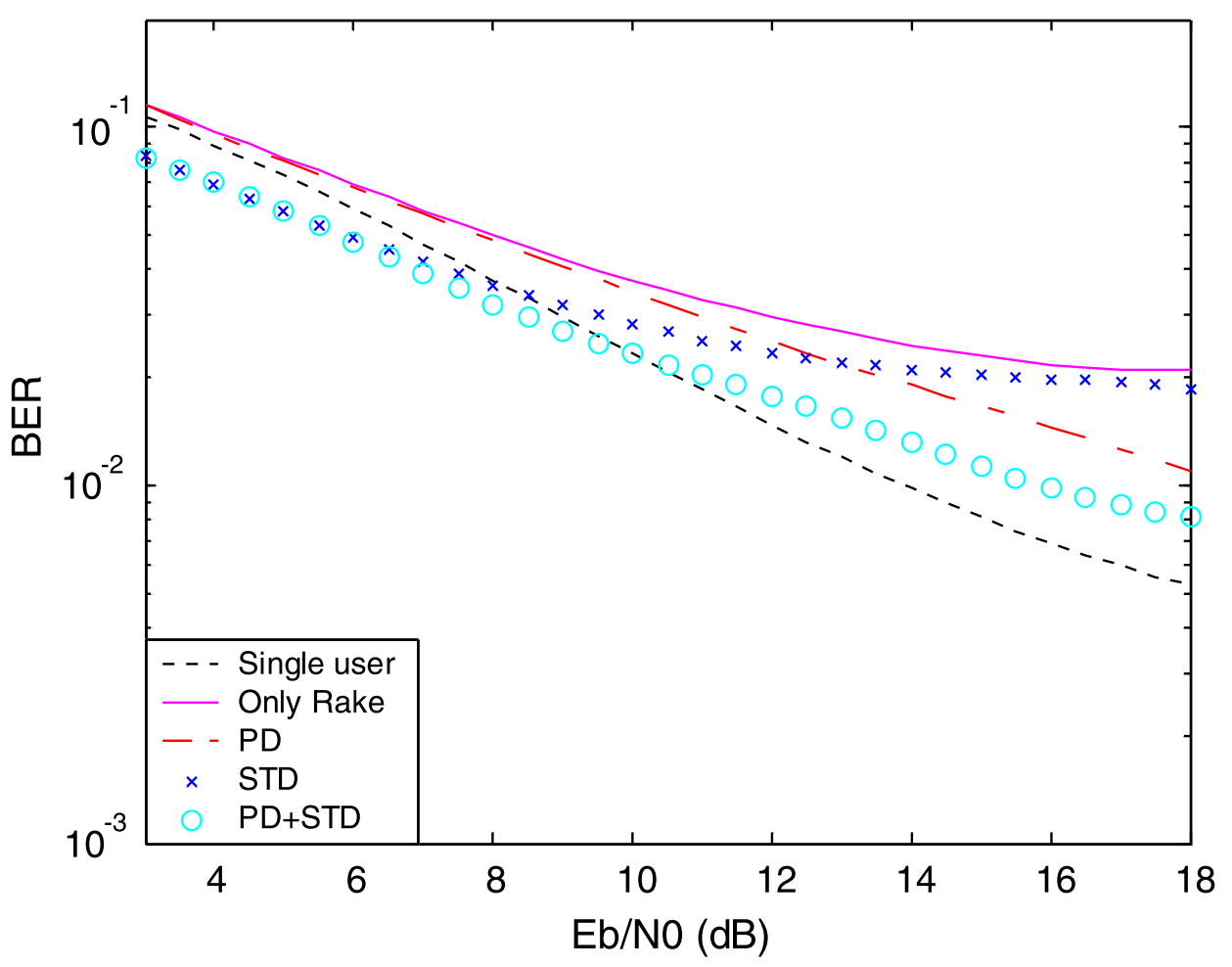

Fig. 6 BER performance for Vehicular A propagation model with 4 interfering users

scheme for this environment is higher than that achieved with in Fig. 4 (Pedestrian A propagation environment). This happens because the Pedestrian A profile is closer to the single path propagation model, and therefore, the multipath able of producing MAI is less intense than that of the Vehicular A propagation model. In other words, the Vehicular A produces higher level of MAI, due to higher power of IPI vectors, than that of the Pedestrian A, and so, the gain obtained with the PD scheme is also increased. It is worth noting that in the downlink, the transmissions to the different users are synchronized. Since Walsh-Hadamard spreading sequences (which are orthogonal) were considered, the MAI is only generated by the multipath propagation channel.

By comparing Fig. 6 with Fig. 4, it can be observed that the performance improvement achieved with the STD relating to "Only Rake" is much higher for the Pedestrian A propagation model than for the Vehicular A propagation model. This happens because the STD tends to achieve better results for propagation models closer to the single-path channel profile [11], while the Alamouti Space-Time Coding tends to perform better in the presence of environments closer to the uniform power profile [12]. Nevertheless, the proposed PD scheme was not properly designed to work with the Alamouti Space-Time Coding, due to its specific decoding algorithm, but with the Rake in the receiver (which allows the combination of the STD scheme without any modification). In any case, the scheme that achieves the best overall performance is the combination of STD with the PD scheme (PD+STD), for higher levels of $E_{b} / N_{0}$. In addition, it can be seen that the "Single user" performance tends to be even better than the PD+STD for $E_{b} / N_{0} \geq 10 \mathrm{~dB}$ because the level of MAI generated by the Vehicular A propagation model is extremely high, even for 4 users, due to the high level of multipath interference.

In Fig. 7 it is shown the performance obtained with 15 users, for the Vehicular A propagation model of 3GPP. Once again, the gain achieved with the STD for the Vehicular A propagation model of 3 GPP with 15 users is lower than that obtained with STD shown in 


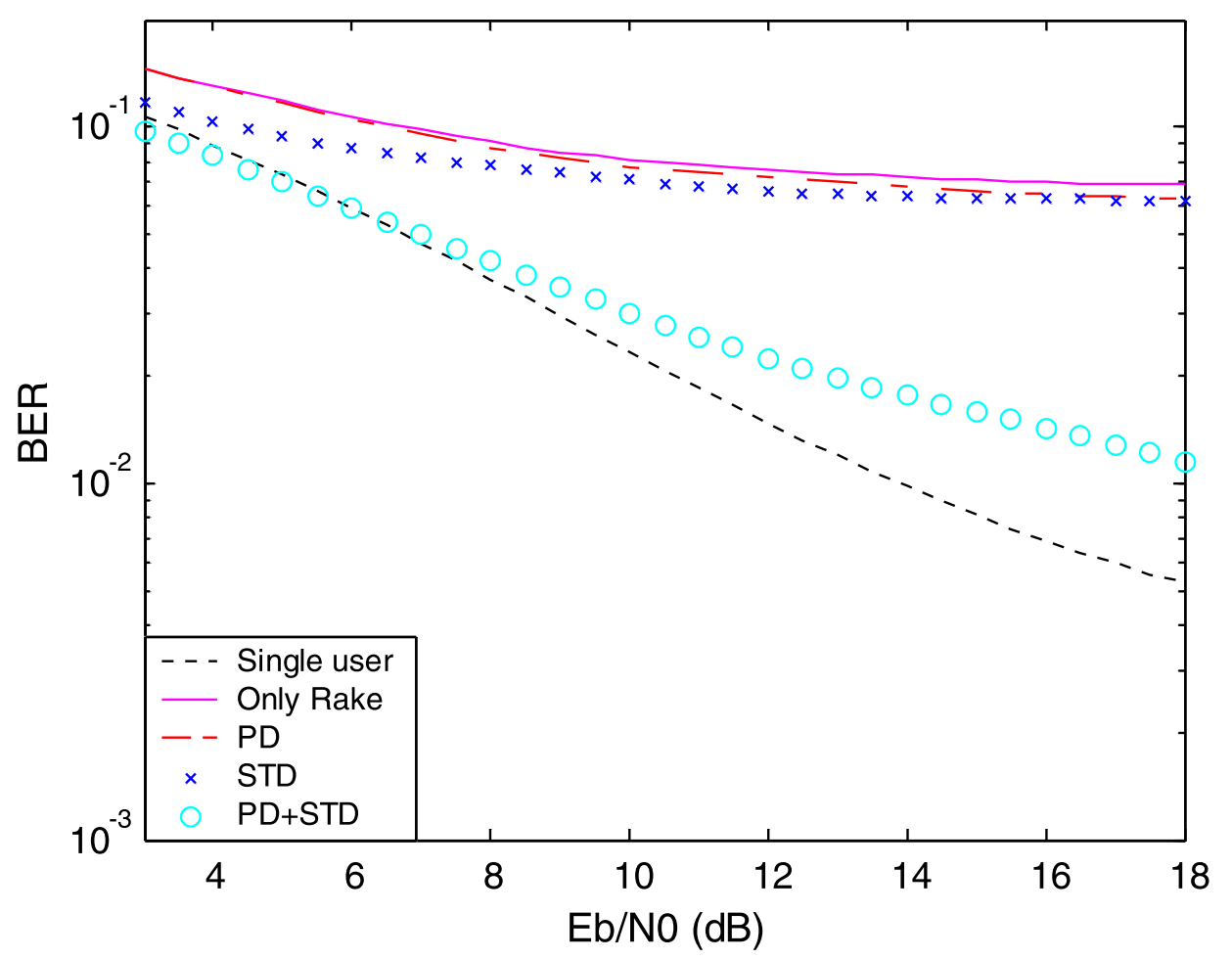

Fig. 7 BER performance for Vehicular A propagation model with 15 interfering users

Fig. 5 (Pedestrian A). As before, the best overall performance is achieved with the combination of the PD with the STD. It can also be seen that the "Single user" performance tends to be even better than the PD + STD for $E_{b} / N_{0} \geq 5 \mathrm{~dB}$ because the level of MAI generated by the Vehicular A propagation model is extremely high. In this case, the "Single user" curve can almost be seen as a lower bound.

\section{Conclusions}

This paper exposed a pre-processing scheme that allows the transfer of the downlink complexity from the receiver (MS) into the transmitter (BS), while keeping the ability to exploit the multipath diversity typical of W-CDMA frequency-selective fading channels, by considering a Rake in the receiver. The proposed scheme can be seen as a simple alternative to MIMO systems or beamforming, presenting a much lower space requirement from the MS (due to the use of a single antenna), and therefore, with an easier practical implementation. Additionally, it is performed without any complexity cost at the MS side (related to the 3GPP Rake receiver).

As a key finding of the proposed PD scheme it can be stated that it tends to achieve a performance improvement over the scheme without PD, especially when combined with the STD, while providing the ability to exploit multipath diversity. The combination of the PD scheme with the STD diversity technique outperforms the component schemes. This happens because, with the combination of both schemes, on the one side, the system is cleaner of MAI, once the orthogonality between the signals seen by the different users can be improved (PD scheme) and, on the other hand, the STD combats the fading.

With the use of the PD scheme we have changed each spreading sequence of each user by a new spreading sequence, which takes into account the several CIRs, in order to minimize the total sum of MAI. 
The performance of the proposed scheme with imperfect downlink channel knowledge at the transmitter side will be considered as a future work.

Acknowledgements This work has been partially funded by the IST-2003-507607 EU Broadcasting and Multicasting Over Enhanced UMTS Mobile Broadband Networks project.

\section{References}

1. Silva, M. M., \& Correia, A. (2003). Joint multi-user detection and intersymbol interference cancellation for WCDMA satellite UMTS. Internation Jounal of Satellite Communications and Networking - Special Issue on Interference Cancellation, 21, 93-117, Wiley-invited paper.

2. Silva, M. M., \& Correia, A. (2000). MAI cancellation with commutation signaling. Proceedings of IEEE VTC'00, Tokyo, Japan, Spring 2000.

3. Silva, M. M., Correia, A., \& Dinis, R. (2005) A decorrelating MUD approach for the downlink of UMTS considering a rake in the receiver - PIMRC 2005, Berlin, Germany, September 2005.

4. Glisic, S., \& Vucetic, B. (1997). Spread spectrum CDMA systems for wireless communications. Artech House Publishers.

5. Choi, J. (2002). Interference mitigation using transmitter filters in CDMA systems. IEEE Transactions on Vehicular Technology, 51(4), 657-666.

6. Vojcic, B. R., \& Jang, W. M. (1998). Transmitter precoding in synchronous multiuser communications. IEEE Transactions on Communications, 46(10), 1346-1355.

7. 3GPP, 25.201-310-Physical Layer General Description.

8. 3GPP, 25.211-370-Physical Channels and mapping of Transport Channels onto Physical Channels.

9. Ding, Y., Davidson, T. N., Luo, Z. Q., \& Wong, K. M. (2003). Minimum BER block precoders for zero-forcing equalization. IEEE Transaction on Signal Processing, 51(9), 2410-2423.

10. Hottinen, A., Tirkkonen, O., \& Wichman, R. (2003). Multi-antenna transceiver techniques for $3 G$ and behing. (Wiley ed.). England.

11. Silva, M. M., \& Correia, A. (2002). Space time coding schemes for 4 or more antennas. Proceedings of IEEE PIMRC'02, Lisbon, Portugal, September 2002.

12. Correia, A. (2003). Transmit diversity schemes for WCDMA. Journal of Wireless Personal Communications, 25, 64-85.

\section{Author Biographies}

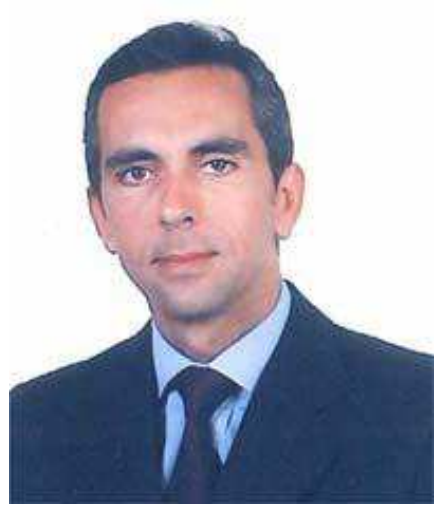

Mário Marques da Silva [marques.silva@ieee.org] is a researcher at the Portuguese Instituto de Telecomunicações. He is also with NATO Air Command Control \& Management Agency (NACMA) in Brussels (Belgium), where he manages the deployable communications of the new Air Command and Control System Program. He received his BSc in Electrical Engineering in 1992, and the MSc and the PhD degrees in Electrical Engineering/Mobile Communications, respectively in 1999 and 2005 from the Instituto Superior Técnico (IST), Technical University of Lisbon, Portugal. He has been leading and cooperating in several telecommunications projects, including research, architecture, development, analysis, simulation and testing of networking, HF, V/UHF, satellite and cellular/UMTS communications systems. He is the author of more than 30 papers and his research interests include several areas of Mobile Communications, namely WCDMA, Multi-User Detection,

Interference Cancellation, Space-Time Coding, MIMO systems, Smart and Adaptive Antennas, Software Defined Radio and Mobile Ad-hoc network. He is a member of IEEE communications society and reviewer of several international publications. 

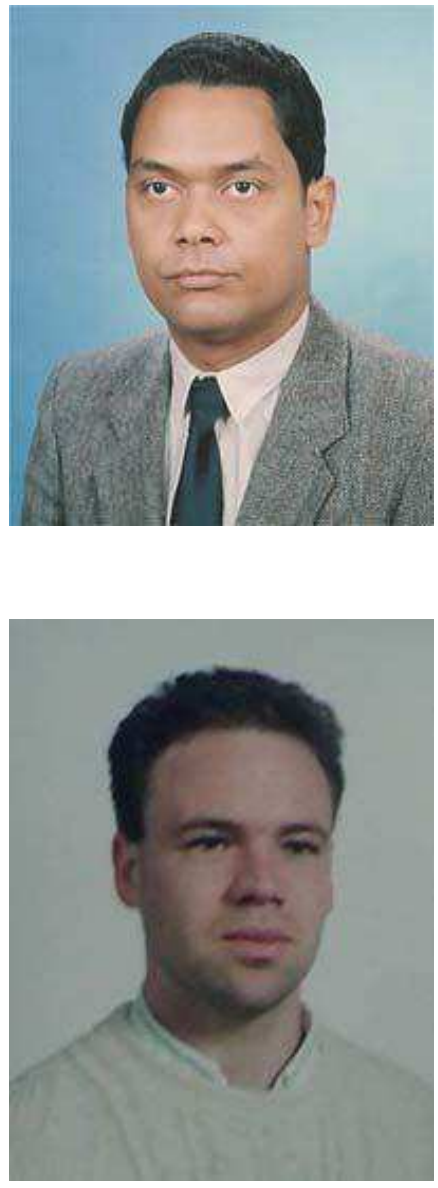

Américo M. C. Correia [americo.correia@iscte.pt] received the BSc degree in Electrical Engineering from the University of Angola in 1983, the MSc. and PhD degrees from Instituto Superior Técnico (IST), Lisbon, Portugal, in 1990 and 1994, respectively. From 1991 to 1999 he was with IST as an Assistant Professor. He is currently with Instituto Superior de Ciências do Trabalho e da Empresa (ISCTE), Lisbon, Portugal. He visited Nokia Research Center from September to December 1998 as a visiting scientist. From September 2000 to August 2001 he joined Ericsson Eurolab Netherlands. His current research topics include, wideband CDMA, MIMO, radio resource management and multimedia broadcast/multicast services.

Rui Dinis [rdinis_home@netcabo.pt] received the $\mathrm{PhD}$ degree from Instituto Superior Técnico (IST), Technical University of Lisbon, Portugal, in 2001. Since 2001 he has been a Professor at IST. He was a member of the research center CAPS/IST (Centro de Análise e Processamento de Sinais) from 1992 to 2001. Since 2002 he is a member of the research center ISR/IST (Instituto de Sistemas e Robótica). He has been involved in several research projects in the broadband wireless communications area. His main research interests include modulation, equalization and channel coding. 